\title{
Assessment of Awareness and Use of Rubber Dam among Dental Practitioners in Navi Mumbai, Maharashtra, India
}

\author{
Sayali Dhamne ${ }^{1}$, Sabita M Ram², Vaibhav P Thakkar ${ }^{3}$, Sanjana S Dharadhar ${ }^{4}$, Ratan G Upadhyay ${ }^{5}$, Tanvi A Patel ${ }^{6}$
}

\begin{abstract}
Aim: This study was planned to assess the awareness and use of rubber dam in routine practice among the dental practitioners of Navi Mumbai, India.

Materials and methods: A Google document with a self-administered validated questionnaire consisting of 14 items was digitally sent to 300 dental practitioners via WhatsApp/e-mail and their responses were collected.

Results: The surveyed responses revealed that 261 (88.2\%) dental practitioners do not use rubber dam in their routine practice, whereas only $35(11.8 \%)$ use it. However, about 166 (58.7\%) dentists showed willingness to inculcate the use of rubber dam in their routine practice after appropriate training and knowledge, while $99(35 \%)$ dentists were not sure of using it and $18(6.4 \%)$ were not keen on using it at all. Moreover, 204 (72.1\%) dental practitioners expressed interest in enhancing their knowledge regarding rubber dam.

Conclusion: The results of this questionnaire-based research study revealed that the prevalence of use and awareness of rubber dam was very limited among the dental practitioners in Navi Mumbai, Maharashtra, India.

Clinical significance: More emphasis should be given to the importance of rubber dam. Various continued dental education (CDE) programs and hands-on workshop can be conducted for dental practitioners.

Keywords: Awareness, Dentistry, Isolation, Rubber dam.

Journal of Contemporary Dentistry (2020): 10.5005/jp-journals-10031-1263
\end{abstract}

\section{INTRODUCTION}

Any dental operative procedure necessitates the need for adequate control over the operating field. It is imperative to have proper moisture control, good accessibility, and visibility as well as adequate room for instruments around the working area. Success of most restorative procedures is dependent on the isolation techniques followed. Such environment is necessary for easy manipulation and insertion of restorative materials. Isolating the working area includes isolation from moisture such as saliva, blood, and gingival sulcular fluid and isolation from the soft tissues such as lips, cheeks, gingiva, and tongue. The goals therefore for isolation in dentistry are moisture control, retraction and access, and harm prevention. These are achieved by either direct or indirect methods. Direct methods include techniques such as rubber dams, cotton rolls and absorbent wafers, evacuator system and saliva ejectors, gingival retraction cord, and mouth props; whereas indirect methods include local anesthesia and drugs such as antianxiety, muscle relaxants, etc. ${ }^{1}$

Rubber dam is a small latex or nonlatex sheet used to isolate a single tooth or multiple teeth from the oral environment for preventing the migration of fluids or foreign objects into or out of the operative field. It is a universally acknowledged mandatory method of isolation particularly during the operative and endodontic procedures. It has several advantages such as it provides adequate access and visibility in the operating field, maximum isolation, creates an aseptic working area, prevents ingestion or aspiration of burs or other instruments, prevents ingestion of irrigants, helps in protection and retraction of soft tissues, and improves infection control. ${ }^{2}$ \begin{tabular}{l}
\hline${ }^{1,3-5}$ Department of Public Health Dentistry, MGM Dental College and \\
Hospital, Navi Mumbai, Maharashtra, India \\
${ }^{2}$ Department of Prosthodontics, MGM Dental College and Hospital, \\
Navi Mumbai, Maharashtra, India \\
${ }^{6}$ Department of Oral and Maxillofacial Pathology, MGM Dental College \\
and Hospital, Navi Mumbai, Maharashtra, India
\end{tabular}

Corresponding Author: Sayali Dhamne, Department of Public Health Dentistry, MGM Dental College and Hospital, Navi Mumbai, Maharashtra, India, Phone: +91 9769596013, e-mail: sayali.dhamne@ gmail.com

How to cite this article: Dhamne S, Ram SM, Thakkar VP, et al. Assessment of Awareness and Use of Rubber Dam among Dental Practitioners in Navi Mumbai, Maharashtra, India. J Contemp Dent 2020;10(1):1-5.

Source of support: Nil

Conflict of interest: None

Despite all these advantages in favor of rubber dam, many dental practitioners fail to use it in their routine practice. ${ }^{3}$ They claim that it is time-consuming, expensive, and uncomfortable for patients. A study by Udoye and Jafarzadeh reported though $77 \%$ of dentists were aware of rubber dam, only $18 \%$ of dentists in a subpopulation of Nigeria use rubber dam. ${ }^{4}$ Also, in a study conducted by Lynch and McConnell on Irish general dental practitioners, it was seen that $57 \%$ of dentists found rubber dam difficult to place and a cumbersome procedure. ${ }^{5}$

The main aim of this study is to assess the awareness regarding rubber dam among the dental practitioners in Navi Mumbai, 
Maharashtra, India, and their willingness to adopt it in their routine practice.

\section{Materials and Methods}

After the ethical approval from Institutional Ethical Review Committee (IERC) to conduct this study, a self-administered validated questionnaire consisting of 14 multiple-choice questions (Table 1) was digitally circulated to dental practitioners in Navi Mumbai via WhatsApp messenger (a texting application). Their responses were collected using a custom Google form. The participating dentists were asked to anonymously answer the questions relevant to them. Informed consent was obtained from the participants before administering the questionnaire. The consent was included as a part of the questionnaire that was circulated. Only dental practitioners registered under the State Dental Register and with minimum of 3 years of dental practice were included in the study. The reason behind this inclusion criteria was to include practitioners who had substantial experience and could respond accurately. All undergraduate dental students and interns were excluded.

To calculate the sample size for the present study, the following formula was used.

$$
\text { Sample size } \begin{aligned}
n= & {[\operatorname{DEFF} \times N p(1-p)]\left[\left(d^{2} / Z_{1-\alpha / 2}^{2}\right) \times(N-1)\right] } \\
& +p \times(1-p)]
\end{aligned}
$$

Population size (for finite population correction factor or $\mathrm{fpc}$ ) $(N): 1,000$

Hypothesized percentage frequency of outcome factor in the population (p): $50 \pm 5 \%$

Confidence limits as percentage of 100 (absolute $\pm \%$ ) (d): $5 \%$

Design effect [for cluster surveys-degrees of freedom (DEFF)]: 1

Based on the above formula, a sample size of 278 was derived. However, an additional $10 \%$ were included in the study $[N=305.8$ (rounded down to 300)] to compensate for potential refusals. The sample size of this study for a subpopulation of Navi Mumbai, Maharashtra, India, was thus estimated to be 300 at $95 \%$ confidence interval.

Questionnaire (Table 1).

\section{Results}

A total of 300 responses were received. Of which 295 (98.3\%) dentists were willing to participate in the study and 5 (1.6\%) refused to participate. Of the 295 dentists who participated in the study, 212 (71.9\%) were general practitioners and 83 (28.1\%) were specialists (Fig. 1).

When asked whether they were taught about rubber dam in their dental graduate study, 205 (69.3\%) denied while 91 (30.7\%) agreed. The survey also showed that 261 (88.2\%) dental practitioners did not use rubber dam in their routine practice, whereas $35(11.8 \%)$ did use (Fig. 2). Among the users of rubber dam, 34 (13.8\%) use it for endodontic procedures, 13 (5.3\%) use it for operative procedures, 6 (2.4\%) use it for pediatric procedures, and 7 (2.8\%) use it for tooth preparation procedures in prosthodontics. $n$ alarming 206 (83.7\%) dentists said they do not use rubber dam for any procedures (Fig. 3).

When surveyed about the most difficult aspect of using rubber dam, 166 (61\%) responded saying selection and adaptation of correct clamp, 51 (18.8\%) said placement of rubber dam, 48 (17.6\%) said placement of the frame, $5(1.9 \%)$ said they have never used it
Table 1: Questionnaire consisting of 14 multiple-choice questions

Questionnaire of rubber dam among the dental practitioners in Navi Mumbai. The information thus obtained will be used only for the research purpose. Participation in the study is entirely on the will of the dentist. Participating dentist's identity will not be revealed and full confidentiality will be assured. No charges will be applicable for the participation in the study. Do you agree to participate in the study? Yes

No

2. Are you a general practitioner or a specialist? General practitioner

Specialist

3. If specialist, please specify your field of specialization

4. Registration number

5. Were you taught placement of rubber dam in your college? Yes

No

6. Do you use rubber dam in your routine practice? Yes

No

7. If yes, for which procedures do you use rubber dam? Endodontic procedures

Operative procedures

Pediatric patients

Tooth preparation procedures for prosthesis

I do not use rubber dam for any procedures

Others

If others, please specify

8. According to you, which is the most difficult aspect about rubber dam procedure?

Placement of the frame

Placement of rubber dam

Selection and adaptation of correct clamp

Other

If others, please specify

9. What in your opinion is the greatest advantage of rubber dam? Provides maximum isolation and aseptic working space

Prevents swallowing or aspiration of instruments/prosthesis

Prevents ingestion of irrigants/other harmful materials used in dentistry

Other

If others, please specify

10. What in your opinion is the greatest disadvantage of rubber dam?

Difficulty in placement

Consumption of time

Expensive

Patient compliance

Other

If others, please specify

11. If not rubber dam which is the other method of isolation that you use in your routine practice?

Cotton rolls

Contd... 
Contd...

\begin{tabular}{l}
\hline Questionnaire \\
\hline Saliva ejectors \\
Cellulose wafers \\
Retraction cords \\
Gauze and throat shields \\
Other \\
If others, please specify \\
12. Do you think there should be more emphasis and proper \\
training in rubber dam usage in dental colleges? \\
Yes \\
No \\
Maybe
\end{tabular}

13. If provided with proper knowledge and training to effectively use rubber dam, would you choose to use it in your routine practice?

Yes

No

Maybe

14. If given an opportunity, are you willing to attend a CDE program or hands-on workshop on rubber dam?

Yes

No

Maybe

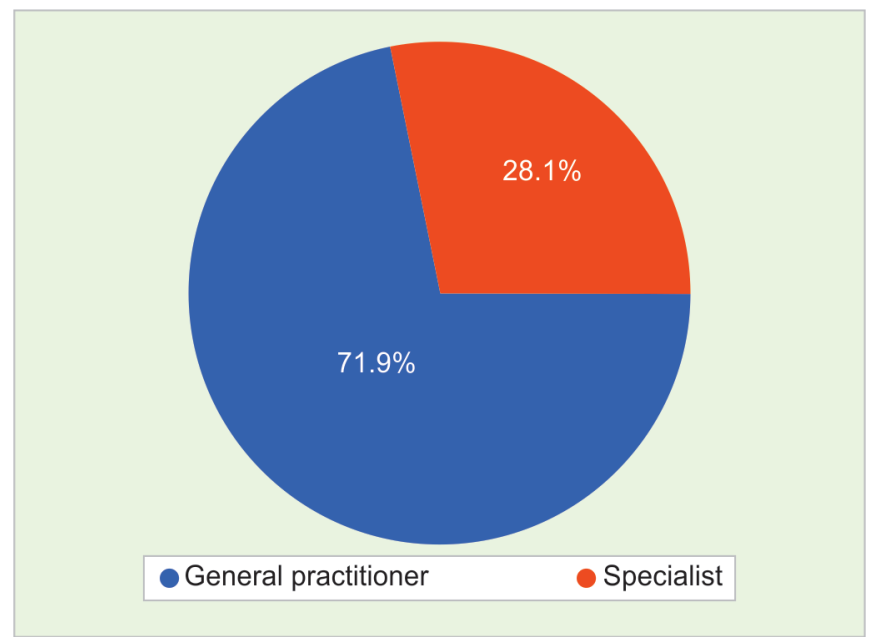

Fig. 1: Distribution of dental practitioners

therefore have no idea, and 1 (0.4\%) said insertion through tight contact (Fig. 4). Dentists were then questioned about their opinion on what they feel is the greatest advantage and disadvantage of rubber dam. In case of advantages, 202 (69.2\%) were of the opinion that it provides maximum isolation and aseptic working area, $67(22.9 \%)$ agreed that it prevents swallowing or aspiration of instruments/prosthesis, and 22 (7.5\%) believed that it prevents ingestion of irrigants and other harmful materials used in dentistry. In case of disadvantages, 116 (39.9\%) believed that it is timeconsuming, $86(29.6 \%)$ were of the opinion that it is difficult to place, 54 (18.6\%) were of the opinion that it is uncomfortable for the patient, and 32 (11\%) were of the opinion that it was expensive.

We also learned of alternatives that were being used in place of rubber dam, such as 267 (90.5\%) preferred saliva ejectors,

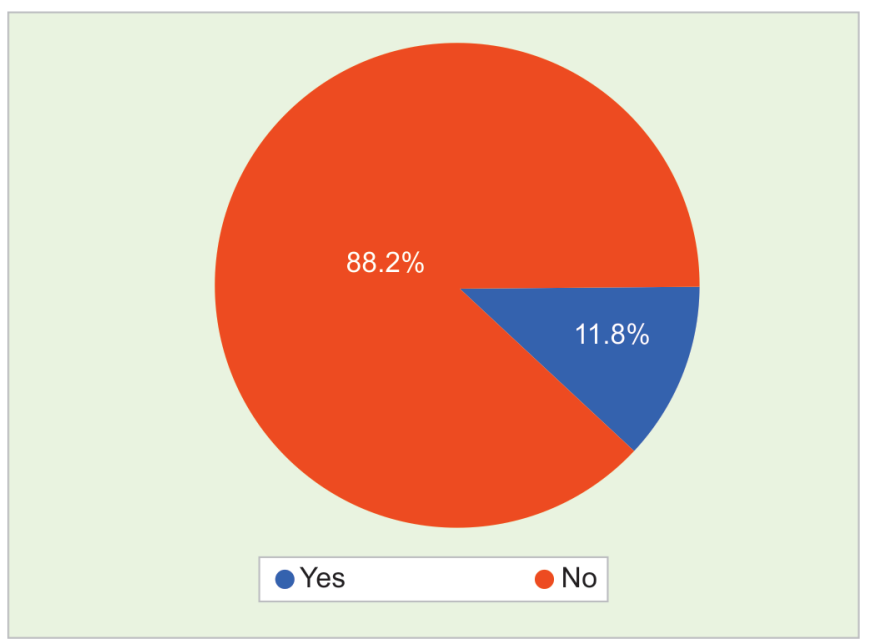

Fig. 2: Number of dental practitioners using rubber dam in routine practice

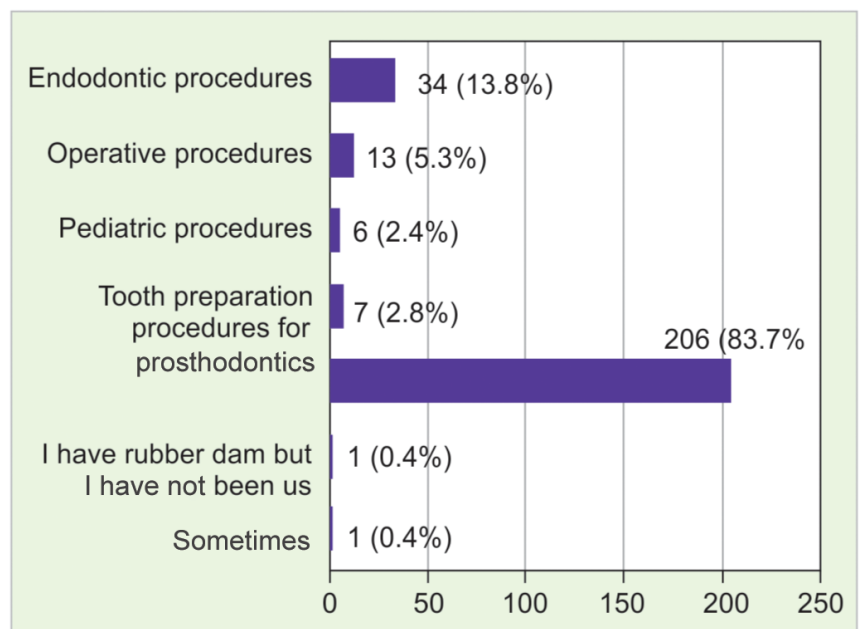

Fig. 3:Various procedures for which dental practitioners use rubber dam

$247(83.7 \%)$ dentists used cotton rolls, 35 (11.9\%) liked retraction chords, 24 (8.1\%) used gauze and throat shields, and 12 (4.4\%) use cellulose wafers as the preferred method of isolation in their practice. We also found that only a few (9) dentists used modified and advanced forms of rubber dam such as minidams and easy dam.

Remarkably, 277 (93.3\%) dentists believed in placing more emphasis and appropriate training in the usage of rubber dam during the graduation, whereas $20(6.7 \%)$ were not sure of this. About 166 (58.7\%) dentists were ready to inculcate utilizing rubber dam in their routine practice with appropriate training and knowledge, 99 (35\%) were not sure and little reluctant in using it, and 18 (6.4\%) were simply not interested (Fig. 5). Two hundred and four $(72.1 \%)$ dental practitioners wanted to enhance their knowledge regarding rubber dam and expressed willingness to attend a related continued dental education (CDE) program or hands-on workshop, 60 (21.2\%) were little reluctant and not sure, and merely 20 (7.1\%) did not show any interest (Fig. 6).

\section{Discussion}

Rubber dam has been universally acknowledged and accepted as the ultimate method of isolation, considering several advantages favoring its use. It has numerous advantages such as it provides adequate access and visibility in the operating field, maximum 


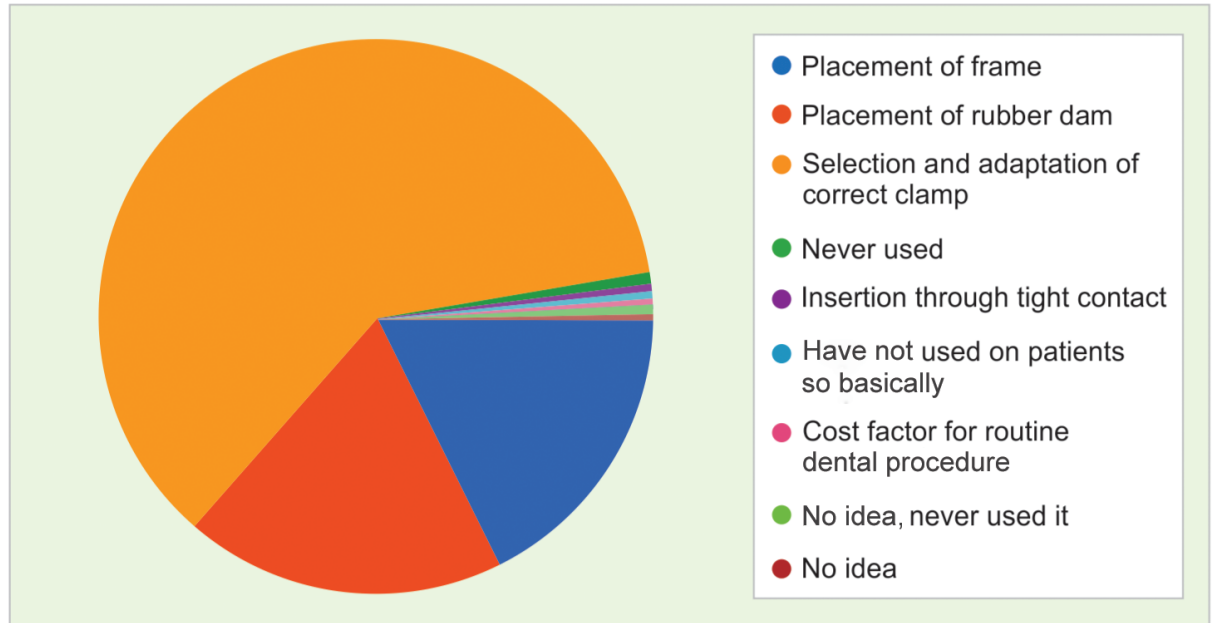

Fig. 4: Difficulties faced by dental practitioners while using rubber dam

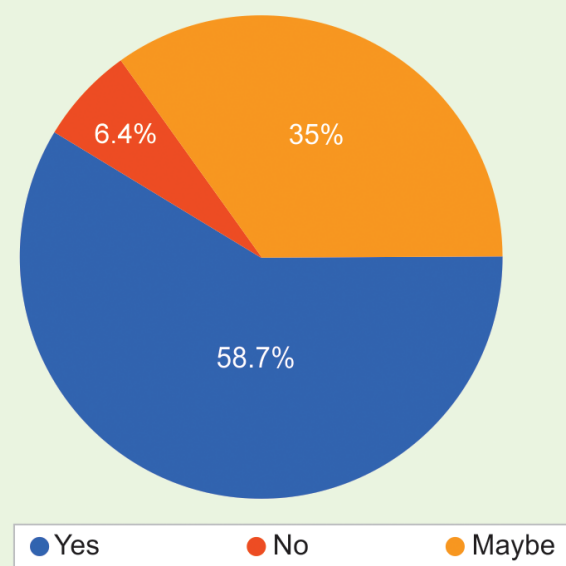

Fig. 5: Number of dentists who are willing to use rubber dam after proper training and knowledge

isolation, creates an aseptic working area, prevents ingestion or aspiration of burs or other instruments, prevents ingestion of irrigants, helps in protection and retraction of soft tissues, and improves infection control. ${ }^{1,2}$ However, its prevalence of use and awareness is low among the dental practitioners. Hence, this study was conducted to learn about this issue among a subpopulation of practicing dentists in Navi Mumbai, Maharashtra, India. Of the 307 dentists who responded, $71.9 \%$ were general practitioners and $28.1 \%$ were specialists. The motive behind asking the field of specialization was to derive a correlation between its major use in specialties such as conservative dentistry and endodontics, pedodontics, and prosthodontics and the awareness of the respective respondents. Having been trained extensively in their specialized field for additional 3 years, we assumed their awareness would be relatively more as compared to the general dental practitioners.

When questioned on if they use rubber dam in their routine practice, about $88.2 \%$ dentists denied and only $35 \%$ were affirmative. On questioning the reasons of difficulty in using rubber dam, $61 \%$ said they find selection and adaptation of correct clamp difficult, $17.6 \%$ said placement of frame being the difficult part, and

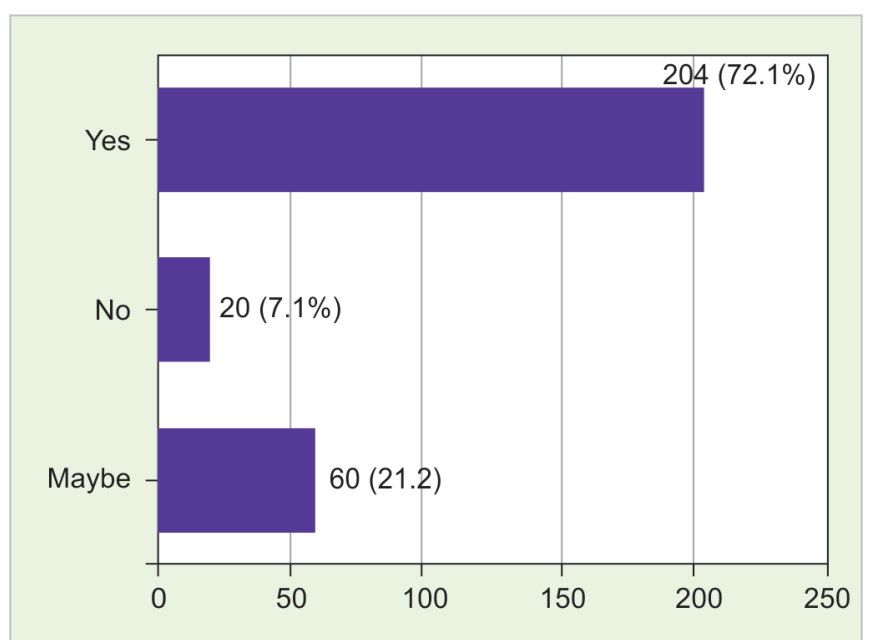

Fig. 6: Number of dental practitioners willing to attend CDE programs and hands-on workshops on rubber dam

$18.8 \%$ said the whole placement of rubber dam is difficult. The main reason behind this scenario and the difficulties faced by the practitioners is the lack of proper training and knowledge during their dental degree curriculum. This was confirmed when $69.3 \%$ dentists complained that they were not taught the use of rubber dam in their dental degree training. A study by Hill and Rubel in USA stated that there is a discrepancy between what is taught at the undergraduate level and general practice of dentistry. ${ }^{3}$ If a proper practical training, rather than just theoretical mention, is included at the level of undergraduation, the situation might change; $93.3 \%$ dentists agreed with this finding. The limited use of rubber dam in our study is similar to what was observed in a study conducted by Udoye and Jafarzadeh in which though $77 \%$ of dentists were aware of rubber dam, only $18 \%$ of dentists in a subpopulation of Nigeria use rubber dam. ${ }^{4}$ Also, in a study conducted by Lynch and McConnell on Irish general dental practitioners, they observed that $57 \%$ of dentists found rubber dam difficult to place and a cumbersome procedure. ${ }^{5}$ A number of other studies were conducted which support a similar claim. ${ }^{6}$

About $39.9 \%$ dentists believe that placement of rubber dam consumes a lot of time and $11 \%$ of them think that it is expensive. 
Thus, dentists opt for other methods of isolation such as saliva ejectors $(90.5 \%)$, cotton rolls $(83.7 \%)$, retraction chords $(11.9 \%)$, gauze and throat shields (8.1\%), and cellulose wafers (4.4\%) which are readily available, easy to use, and cost-effective. A surprising result was that a few dentists (merely 1\%) use modified and advanced forms of rubber dams such as minidams and easy dams; $18.6 \%$ of dentists state that the placement of rubber dam provides discomfort to the patient. A study conducted by Stewardson and McHugh wherein they recorded the patients experience on the use of rubber dam in Birmingham dental school and general dental practitioners showed that patients were generally not against the use of rubber dam and operator experience helped with patient compliance. $^{7,8}$

Although dental practitioners agree with the several advantages and benefits of rubber dam, there is a lack of proper training and knowledge. Therefore, $72.1 \%$ dentists are willing to attend CDE programs and hands-on workshop to enhance their knowledge following which $58.7 \%$ dentists are willing to start utilizing dams in their routine practice. However, $21.2 \%$ is little skeptical and reluctant in attending such programs and $35 \%$ in using it, barring $7-8 \%$ who does not want to train and use it at all.

\section{Conclusion}

The prevalence of use and awareness of rubber dam is very limited among the dental practitioners in Navi Mumbai, Maharashtra, India. Although dentists are aware of its numerous advantages and consider it as one of the most effective method of isolation, the rubber dam is underused in this region. It can be inferred from this study that the major reason behind this is lack of proper training and knowledge. However, dentists in this region are willing to train themselves and inculcate the use of rubber dam in their routine practice.

\section{Clinical Significance}

The clinical significance of this study is that there is an urgent need to place more emphasis on rubber dam usage. Awareness can be raised via several CDE programs and also hands-on workshops that can provide appropriate training for dental practitioners and students.

\section{References}

1. Ahmad I. Rubber dam usage for endodontic treatment, a review. Int Endod J 2009;42(11):963-972. DOI: 10.1111/j.1365-2591.2009. 01623.x.

2. Liebenberg WH. Manipulation of rubber dam septa: an aid to the meticulous isolation of splinted prostheses. J Endod 1995;21(4): 208-211. DOI: 10.1016/S0099-2399(06)80568-6.

3. Hill EE, Rubel BS. Do dental educators need to improve their approach to teaching rubber dam use? J Dent Educ 2008;72(10):1177-1181.

4. Udoye $\mathrm{Cl}$, Jafarzadeh $\mathrm{H}$. Rubber dam use among a subpopulation of Nigerian dentists. J Oral Sci 2010;52(2):245-249. DOI: 10.2334/ josnusd.52.245.

5. Lynch CD, McConnell RJ. Attitude and use of rubber dam by Irish general dental practitioners. Int Endod J 2007;40(6):427-432. DOI: 10.1111/j.1365-2591.2007.01212.x.

6. Joynt RB, Davis EL, Schreier PH. Rubber dam usage among practicing dentists. Oper Dent 1989;14(4):176-181.

7. Stewardson DA, McHugh ES. Patients attitude to rubber dam. Int Endod J 2002;35(10):812-819. DOI: 10.1046/j.1365-2591.2002. 00571.x.

8. Jones CM, Reid JS. Patient and operator attitude towards rubber dam. ASDC J Dent Child 1988;55(6):452-454. 